\title{
The Hubbard model at half a century
}

\author{
Models are abundant in virtually all branches of physics, with some achieving iconic status. The Hubbard \\ model, celebrating its golden jubilee this year, continues to be one of the most popular contrivances of \\ theoretical condensed-matter physics.
}

Capturing the essence of a phenomenon while being simple: the ingredients of a top model in physics. Since the early days of quantum mechanics, many models, Hamiltonians and theories aiming to provide a deeper understanding of various properties of condensed matter have been put forward - with varying degrees of success and fame. One truly legendary model is the Hubbard model, independently conceived by Martin Gutzwiller ${ }^{1}$, Junjiro Kanamori ${ }^{2}$ and, of course, John Hubbard ${ }^{3}$ - their original papers all appearing in 1963. The main motivation was the need for a way to tackle the behaviour of correlated (rather than non-interacting) electrons in solids. Initially, the model was introduced to provide an explanation for the itinerant ferromagnetism of transition metals, such as iron and nickel, but the past 50 years have seen its relevance go far beyond that original context.

Technically, the Hubbard model is an extension of the so-called tight-binding model, wherein electrons can hop between lattice sites without 'feeling' each other. In its simplest form, electron hopping can only take place between nearest-neighbour sites, and all hopping processes have the same kinetic energy, $-t$. The tight-binding model evinces the quantum-mechanical quintessence of electrons in a solid: the emergence of an electronic band structure intervals of allowed and forbidden energies - lying at the heart of present-day semiconductor technology.

Hubbard's Hamiltonian features an additional term, introducing an energy amount $U$ for each pair of electrons occupying the same lattice site - representing Coulomb repulsion. Hubbard found the model to be the simplest that produces both a metallic and an insulating state, depending on the value of $U$. (Incidentally, the commonly known 'Hubbard $U$ ' was actually called ' $I$ ' by Hubbard, following John Slater's notation used in a precursor to the model. Philip Anderson seems to be the first one to have used ' $U$ '.)

Following the submission of his 1963 paper $^{3}$, Hubbard continued to improve and refine his model. His 'Electron correlations in narrow energy bands' would eventually comprise six installments. 'Hubbard III'4 became especially important as it showed that for one electron per lattice site - the Hubbard model at half filling - the Mott (or Mott-Hubbard) transition is reproduced. This is a type of metal-insulator transition that could not be understood in terms of conventional band theory (which predicts that a half-filled band always results in a conducting state).

The simplicity of the Hubbard model, when written down, is deceptive. Not only had Gutzwiller, Kanamori and Hubbard already extracted different physics from the model, it turned out to be a 'mathematically hard' problem: an exact solution has so far only been obtained for the one-dimensional case. Today, with ever-increasing computer power, numerical simulations of the model are mainstream — particularly when trying to get a grip on the role of the topology of the underlying lattice, a 'hidden variable indeed.

Ever since its inception, the model has spawned new lines of research in theoretical physics; the development of dynamical mean-field theory is a noteworthy example. Although the model quickly became a firm favourite of theorists, it twice experienced a sudden rise in popularity due to breakthroughs in experimental physics.

The first followed the discovery of high-temperature superconductors in 1986. Until then, the Hubbard model was believed to have little to do with superconductivity. However, in the wake of the high-temperature superconductivity 'revolution', one particular adaptation of Hubbard's original model called the $t-J$ model (originally arising in the context of doped Mott-Hubbard insulators) emerged as a compelling candidate for hosting a superconducting state. A rigorous proof of the existence (or non-existence) of a superconducting ground state in the $t-J$ model is still missing - underlining that research on superconductivity and the Hubbard model is continuing.

A second boost in activity surrounding the Hubbard model came in the 2000s, when the field of cold-atom optical trapping had advanced so far that experimental realizations of the Hubbard model could be achieved. A landmark experiment demonstrated how a lattice of bosonic atoms displays a transition from a superfluid to a Mott insulator ${ }^{5}$, a result accounted for by the Bose-Hubbard model (the Hubbard model for bosons). Many other variants of the Hubbard model, including the original model for fermions ${ }^{6}$, have been experimentally realized by now, a development that nicely illustrates how a model can become the target of experiments itself - and, more generally, how theoretical and experimental physics can entangle and spark further progress.

\section{The simplicity of the Hubbard model, when written down, is deceptive.}

Part of the legacy of Hubbard's model is that it launched the field of strongly correlated systems - it is undoubtedly the archetypal model of many-body physics. (As an aside, when asked by his motherin-law what the book he was reading The Many-Body Problem - was about, John Hubbard replied, without even the faintest attempt at a serious explanation: "A murder mystery.")

Although the Hubbard model secured it's place in (condensed-matter) physics textbooks many decades ago, it is very likely that it will continue to play an important role in fundamental research as well. In particular, the continuing experimental progress in artificial lattices of cold atoms and superconductivity, where the Hubbard model and its modifications play a prominent role, should be a stimulus for further explorations.

\footnotetext{
References

1. Gutzwiller, M. C. Phys. Rev. Lett. 10, 159-162 (1963)

2. Kanamori, J. Prog. Theor. Phys. 30, 275-289 (1963).

3. Hubbard, J. Proc. R. Soc. A 276, 237-257 (1963).

4. Hubbard, J. Proc. R. Soc. A 281, 401-419 (1964).

5. Greiner, M., Mandel, O., Esslinger, T., Hänsch, T. W. \& Bloch, I. Nature 415, 39-44 (2002).

6. Jördens, R., Strohmaier, N., Günter, K., Moritz, H. \& Esslinger, T. Nature 455, 204-207 (2008).
} 